\title{
ON THE LOCAL STRONG SOLUTIONS FOR A SYSTEM DESCRIBING THE FLOW OF A VISCOELASTIC FLUID
}

\author{
ONDŘEJ KREML and MILAN POKORNÝ \\ Mathematical Institute of Charles University \\ Sokolovská 83, 18675 Praha 8, Czech Republic \\ E-mail: kreml@karlin.mff.cuni.cz, pokorny@karlin.mff.cuni.cz
}

\begin{abstract}
We consider a model for the viscoelastic fluid which has recently been studied in [4] and [1]. We show the local-in-time existence of a strong solution to the corresponding system of partial differential equations under less regularity assumptions on the initial data than in the above mentioned papers. The main difference in our approach is the use of the $L^{p}$ theory for the Stokes system.
\end{abstract}

1. Introduction. Biological fluids (blood, synovial fluid), asphalt and some geomaterials (sand, clay) are materials with complicated structure consisting of many components. None the less we can still model them as a continuum if we are interested in the behavior of this material as a whole body. Since these materials consist of many components they have some non-newtonian characteristics such as shear thinning or thickening, stress relaxation and so on. We can describe these properties using models of viscoelastic fluids of the rate type.

We assume that the fluid is incompressible which is for many flow regimes in a good agreement with experimental data. Thus we consider the following system of partial differential equations: ${ }^{1}$

$$
\left.\begin{array}{c}
\frac{\partial \mathbf{v}}{\partial t}+\mathbf{v} \cdot \nabla \mathbf{v}-\operatorname{div} \mathbf{T}=\mathbf{f} \\
\operatorname{div} \mathbf{v}=0
\end{array}\right\} \text { in }(0, T) \times \Omega
$$

where $\mathbf{v}$ is the velocity field, $\mathbf{T}$ the stress tensor and $\mathbf{f}$ the vector of external forces. Note that the constant density is for the sake of simplicity assumed to be equal to one. We

2000 Mathematics Subject Classification: 35Q35, 76D03, 35Q30.

Key words and phrases: viscoelastic fluid, Oldroyd model, local strong solution, $L^{p}$ theory.

The paper is in final form and no version of it will be published elsewhere.

${ }^{1}$ In the whole text we will denote scalars by small letters $(\mu, p, \ldots)$, vectors by bold small letters $(\mathbf{v}, \mathbf{x}, \ldots)$ and tensors by bold capital letters $(\mathbf{T}, \mathbf{F}, \ldots)$. 
consider the stress tensor $\mathbf{T}$ in the form

$$
\mathbf{T}=-p \mathbf{I}+2 \mu \mathbf{D}(\mathbf{v})+\mathbf{T}_{E},
$$

where $p$ is the pressure, $\mathbf{D}(\mathbf{v})$ is the symmetric part of the velocity gradient, $\mu>0$ the viscosity and for the elastic part of the stress tensor $\mathbf{T}_{E}$ we assume

$$
\mathbf{T}_{E}=\frac{\partial S(\mathbf{F})}{\partial \mathbf{F}} \mathbf{F}^{T}
$$

where $S(\mathbf{F})$ is the density of the elastic energy of the material and $\mathbf{F}(t, \mathbf{x})$ denotes the deformation gradient. We will consider only the simplest reasonable case of elasticity, namely $S(\mathbf{F})=|\mathbf{F}|^{2}=\operatorname{tr}\left(\mathbf{F} \mathbf{F}^{T}\right)$ and thus $\mathbf{T}_{E}=\mathbf{F} \mathbf{F}^{T}$. Note that even in this case the elastic part of the stress is a nonlinear function of the deformation gradient. Moreover, by simple calculation, one easily sees that the tensor $\mathbf{F}$ satisfies the following transport equation

$$
\frac{\partial \mathbf{F}}{\partial t}+(\mathbf{v} \cdot \nabla) \mathbf{F}=\nabla \mathbf{v} \mathbf{F}
$$

Therefore the elastic stress tensor $\mathbf{T}_{E}$ fulfills

$$
\frac{\partial \mathbf{T}_{E}}{\partial t}+(\mathbf{v} \cdot \nabla) \mathbf{T}_{E}-\nabla \mathbf{v} \mathbf{T}_{E}-\mathbf{T}_{E}(\nabla \mathbf{v})^{T}=\mathbf{0}
$$

and we recover the upper convected Oldroyd model with some zero coefficients.

Inserting the form of the stress tensor into (1) we get the following system of partial differential equations:

$$
\begin{aligned}
& \left.\begin{array}{c}
\frac{\partial \mathbf{v}}{\partial t}+\mathbf{v} \cdot \nabla \mathbf{v}-\mu \Delta \mathbf{v}+\nabla p=\operatorname{div}\left(\mathbf{F F}^{T}\right)+\mathbf{f} \\
\operatorname{div} \mathbf{v}=0 \\
\frac{\partial \mathbf{F}}{\partial t}+\mathbf{v} \cdot \nabla \mathbf{F}=\nabla \mathbf{v} \mathbf{F}
\end{array}\right\} \text { in }(0, T) \times \Omega \\
& \mathbf{v}(0, \mathbf{x})=\mathbf{v}_{0}(\mathbf{x}) \quad \text { in } \Omega, \\
& \mathbf{F}(0, \mathbf{x})=\mathbf{F}_{0}(\mathbf{x}) \quad \text { in } \Omega,
\end{aligned}
$$

where $\mathbf{v}_{0}(\mathbf{x})$ and $\mathbf{F}_{0}(\mathbf{x})$ are the initial conditions. Note that we use the convention $(\operatorname{div} \mathbf{H})_{j}=\sum_{i=1}^{N} \frac{\partial H_{i j}}{\partial x_{i}}$.

For more details about obtaining this system and its relation to Oldroyd models see [6] and [4].

Taking divergence of $(2)_{3}$ yields

$$
\frac{\partial(\operatorname{div} \mathbf{F})}{\partial t}+(\mathbf{v} \cdot \nabla)(\operatorname{div} \mathbf{F})=0
$$

and therefore assuming $\operatorname{div} \mathbf{F}_{0}=0$ we have $\operatorname{div} \mathbf{F}(t)=0$ for $t>0$.

In two space dimensions we can use this divergence-free condition to rewrite our system. There exists $\phi=\left(\phi_{1}, \phi_{2}\right)$ such that

$$
\mathbf{F}=\left(\begin{array}{cc}
-\frac{\partial \phi_{1}}{\partial x_{2}} & -\frac{\partial \phi_{2}}{\partial x_{2}} \\
\frac{\partial \phi_{1}}{\partial x_{1}} & \frac{\partial \phi_{2}}{\partial x_{1}}
\end{array}\right) .
$$


System (2) can be transformed into

$$
\left.\begin{array}{cc}
\frac{\partial \mathbf{v}}{\partial t}+\mathbf{v} \cdot \nabla \mathbf{v}-\mu \Delta \mathbf{v}+\nabla p=-\sum_{i=1}^{N} \Delta \phi_{i} \nabla \phi_{i}+\mathbf{f} \\
\operatorname{div} \mathbf{v}=0 \\
\frac{\partial \phi}{\partial t}+\mathbf{v} \cdot \nabla \boldsymbol{\phi}=\mathbf{0} \\
\mathbf{v}(0, \mathbf{x})=\mathbf{v}_{0}(\mathbf{x}) & \text { in } \Omega, \\
\boldsymbol{\phi}(0, \mathbf{x})=\boldsymbol{\phi}_{0}(\mathbf{x}) & \text { in } \Omega
\end{array}\right\} \text { in }(0, T) \times \Omega
$$

This system was studied by Lin, Liu and Zhang [4]. The authors proved the existence of smooth solutions on short time intervals and global existence of smooth solutions provided the initial data are sufficiently close to the equilibrium state. The main difficulty to prove these results lies in the free transport equation of $\phi$, which does not show any dissipative mechanism. However, in [4] quite high regularity of the initial conditions, at least $\mathbf{v}_{0} \in W^{2,2}(\Omega)$ and $\phi_{0} \in W^{3,2}(\Omega)$, was required. The authors use the standard technique of $L^{2}$ energy estimates for $\mathbf{v}, \phi$ and their derivatives. Our approach is different, we use the $L^{p}$ theory of the Stokes system to achieve the existence of smooth solutions on short time intervals under less regular initial conditions, namely $\nabla \phi_{0} \in W^{1, p}(\Omega)$ for $p>2$ and $\mathbf{v}_{0} \in W_{\text {div }}^{1,2}(\Omega)$.

In three space dimensions the situation is different. In this case the restriction $\operatorname{div} \mathbf{F}=$ $\mathbf{0}$ does not yield a system like (3). Actually one may obtain a much more complex system if one persists to find such kind of equivalent form. Nevertheless, similar results as in [4] were obtained by Chen and Zhang in [1] for system (2); however, they required at least the same regularity of the initial data as in [4]. Again, the authors use the technique of $L^{2}$ energy estimates for $\mathbf{v}, \mathbf{F}$ and their derivatives. Our approach is the same as in two space dimensions, we use the $L^{p}$ theory of the Stokes system to achieve the existence of smooth solutions on short time intervals under similar initial conditions as in $2 \mathrm{D}$, namely $\mathbf{F}_{0} \in W^{1, p}(\Omega)$ for $p>3$ and $\mathbf{v}_{0} \in W_{\text {div }}^{1,2}(\Omega)$.

Oldroyd models and models related to them are of great interest. P.-L. Lions and N. Masmoudi [5] obtained global existence of weak solutions to the Oldroyd model with the corotational time derivative. Recently, a more complex system (the FENE dumbbell model for polymeric flow) was studied by N. Masmoudi [7], where the Navier-Stokes equation is coupled with a nonlinear Fokker-Planck equation which describes the evolution of the polymer density. The author proves similar statements as in [4] and [1].

In the following text we will use standard notation for Lebesgue, Sobolev and Bochner spaces. We will also use the standard notation for weak $(\rightarrow)$ and strong $(\rightarrow)$ convergence. For $X(\Omega)$ a Banach space, we will write $X$ instead of $X(\Omega)$ if no confusion can arise.

2. Preliminaries. We recall several results concerning the solvability and estimates for Stokes and Navier-Stokes system. First, let us consider the Stokes problem

$$
\left.\begin{array}{c}
\frac{\partial \mathbf{v}}{\partial t}-\mu \Delta \mathbf{v}+\nabla p=\mathbf{f} \\
\operatorname{div} \mathbf{v}=0 \\
\mathbf{v}(0, \mathbf{x})=\mathbf{v}_{0}(\mathbf{x})
\end{array}\right\} \text { in }(0, T) \times \Omega
$$


with either $\Omega=\mathbb{R}^{N}$ (we denote this problem $\left.C_{S}\right), \Omega=(0, L)^{N}$ and periodic boundary conditions (we denote this problem $P_{S}$ ) or $\Omega$ smooth with homogeneous Dirichlet boundary conditions (we denote it $D_{S}$ ).

Let $D_{q}^{1-\frac{1}{s}, s}(\Omega)$ be the space obtained by real interpolation

$$
D_{q}^{1-\frac{1}{s}, s}(\Omega)=\left(L_{\text {div }}^{q}(\Omega), W_{\text {div }}^{2, q}(\Omega)\right)_{1-\frac{1}{s}, s},
$$

where

$$
L_{d i v}^{q}(\Omega)={\overline{\left\{\mathbf{u} \in C_{0}^{\infty}(\Omega), \operatorname{div} \mathbf{u}=0\right\}}}^{\|\cdot\|_{L^{q}}} \quad \text { for } D_{S} \text { and } C_{S},
$$

or

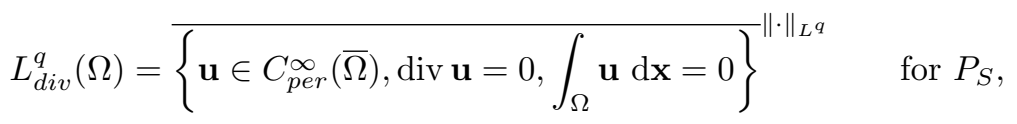

and

$$
W_{\text {div }}^{2, q}(\Omega)=\left\{\mathbf{u} \in W^{2, q}(\Omega) \cap W_{0}^{1, q}(\Omega), \operatorname{div} \mathbf{u}=0\right\} \quad \text { for } D_{S} \text { and } C_{S},
$$

or

$$
W_{\text {div }}^{2, q}(\Omega)=\left\{\mathbf{u} \in W_{\text {per }}^{2, q}(\Omega), \operatorname{div} \mathbf{u}=0, \int_{\Omega} \mathbf{u} \mathrm{d} \mathbf{x}=0\right\} \quad \text { for } P_{S}
$$

We have

Lemma 2.1 (Giga, Sohr). Let $\mathbf{v}_{0} \in D_{p}^{1-1 / q, q}(\Omega), \Omega \in C^{2, \lambda}$ for $\lambda>0$ (for $D_{S}$ ), $\mathbf{f} \in$ $L^{q}\left(0, T, L^{p}(\Omega)\right), 1<p, q<\infty$. Then there exists unique solution to $C_{S}, P_{S}$ or $D_{S}$ and the solution satisfies

$$
\begin{array}{r}
\left\|\nabla^{2} \mathbf{v}\right\|_{L^{q}\left(0, T, L^{p}\right)}+\|\nabla p\|_{L^{q}\left(0, T, L^{p}\right)}+\left\|\frac{\partial \mathbf{v}}{\partial t}\right\|_{L^{q}\left(0, T, L^{p}\right)} \\
\leq C\left(\|\mathbf{f}\|_{L^{q}\left(0, T, L^{p}\right)}+\left\|\mathbf{v}_{0}\right\|_{\left.D_{p}^{1-1 / q, q}\right)} .\right.
\end{array}
$$

The constant $C$ does not depend on $T$.

Proof. See [3].

Next we assume $\mathbf{f}=\operatorname{div} \mathbf{F}$ in (4) with $\mathbf{v}_{0}=0$. The nonzero initial condition may also be treated, however, it is more complicated and we do not need it here. We have

Lemma 2.2 (Giga, Giga, Sohr). Let $\Omega \in C^{2, \lambda}, \lambda>0$ for the problem $D_{S}, 1<p, q<\infty$ and $\mathbf{F} \in L^{q}\left(0, T, L^{p}(\Omega)\right)$. Then there exists a unique weak solution to (4) (either $C_{S}$ or $D_{S}$ or $\left.P_{S}\right)$ with $\mathbf{f}=\operatorname{div} \mathbf{F}$ and $\mathbf{v}_{0}=0$ and it satisfies

$$
\|\nabla \mathbf{v}\|_{L^{q}\left(0, T, L^{p}\right)} \leq C\|\mathbf{F}\|_{L^{q}\left(0, T, L^{p}\right)} .
$$

The constant $C$ does not depend on $T$.

Proof. See [2].

Next we consider the Navier-Stokes problem

$$
\left.\begin{array}{c}
\frac{\partial \mathbf{v}}{\partial t}+\mathbf{v} \cdot \begin{array}{c}
\nabla \mathbf{v}-\mu \Delta \mathbf{v}+\nabla p=\mathbf{f} \\
\operatorname{div} \mathbf{v}=0 \\
\mathbf{v}(0, \mathbf{x})=\mathbf{v}_{0}(\mathbf{x})
\end{array}
\end{array}\right\} \text { in } \Omega,
$$


with the same "boundary" conditions as for the Stokes problem (we denote the corresponding problems by $D_{N S}, P_{N S}$ or $C_{N S}$ as above). We also denote

$$
\begin{array}{cc}
W_{d i v}^{1,2}(\Omega)=\left\{\mathbf{u} \in W_{0}^{1,2}(\Omega), \operatorname{div} \mathbf{u}=0\right\} & \text { for } D_{N S} \text { and } C_{N S}, \\
W_{d i v}^{1,2}(\Omega)=\left\{\mathbf{u} \in W_{\text {per }}^{1,2}(\Omega), \operatorname{div} \mathbf{u}=0, \int_{\Omega} \mathbf{u} d x=\mathbf{0}\right\} & \text { for } P_{N S} .
\end{array}
$$

Concerning the existence, we have the following classical results (see e.g. [8])

Lemma $2.3(N=2)$. Let $\Omega \in C^{2}$ (for $\left.D_{N S}\right), \mathbf{f} \in L^{2}((0, T) \times \Omega), \int_{\Omega} \mathbf{f} d x=\mathbf{0}$ (for $P_{N S}$ ) $\mathbf{v}_{0} \in W_{\text {div }}^{1,2}(\Omega)$. Then there exists a unique global-in-time strong solution to the NavierStokes system (5) such that

$$
\mathbf{v} \in L^{\infty}\left(0, T, W_{d i v}^{1,2}(\Omega)\right) \cap L^{2}\left(0, T, W^{2,2}(\Omega)\right)
$$

and

$$
\frac{\partial \mathbf{v}}{\partial t}, \nabla p \in L^{2}((0, T) \times \Omega)
$$

Lemma $2.4(N=3)$. Let $\Omega$ be an open set, $\mathbf{f} \in L^{2}((0, T) \times \Omega), \int_{\Omega} \mathbf{f} d x=\mathbf{0}$ (for $P_{N S}$ ), $\mathbf{v}_{0} \in W_{\text {div }}^{1,2}(\Omega)$. Then there exists a global-in-time weak solution to the Navier-Stokes system (5) such that

$$
\mathbf{v} \in L^{\infty}\left(0, T, L^{2}(\Omega)\right) \cap L^{2}\left(0, T, W_{d i v}^{1,2}(\Omega)\right)
$$

and

$$
\frac{\partial \mathbf{v}}{\partial t} \in L^{4 / 3}\left((0, T), W^{-1,2}(\Omega)\right) .
$$

Moreover, if $\Omega \in C^{2}$ (for $D_{N S}$ ), there exists $T^{*}>0$ such that in $\left(0, T^{*}\right)$ this solution is strong, unique in the class of all weak solutions satisfying the energy inequality, i.e.

$$
\mathbf{v} \in L^{\infty}\left(0, T^{*}, W_{d i v}^{1,2}(\Omega)\right) \cap L^{2}\left(0, T^{*}, W^{2,2}(\Omega)\right)
$$

and

$$
\frac{\partial \mathbf{v}}{\partial t}, \nabla p \in L^{2}\left(\left(0, T^{*}\right) \times \Omega\right) .
$$

Finally, we need the following version of the Banach fixed-point theorem.

Lemma 2.5. Let $X$ be a reflexive Banach space or let $X$ have a separable pre-dual. Let $H$ be a convex, closed and bounded subset of $X$ and let $X \hookrightarrow Y$, where $Y$ is a Banach space. Let $\mathcal{T}: X \rightarrow X$ map $H$ into $H$ and let

$$
\|\mathcal{T} u-\mathcal{T} v\|_{Y} \leq \rho\|u-v\|_{Y} \quad \forall u, v \in H, \quad \rho<1 .
$$

Then there exists a unique fixed point of $\mathcal{T}$ in $H$.

Proof. Let $u_{0} \in H$ be an arbitrary element. Define $u_{n}=\mathcal{T} u_{n-1} \forall n \in \mathbb{N}$. The operator $\mathcal{T}$ is contraction in $Y$, hence there exists $u \in Y$ such that $u_{n} \rightarrow u$ in $Y$. As $u_{n}$ is bounded in $X$, there exists $\bar{u} \in X$ such that $u_{n} \rightarrow \bar{u}$ (or $u_{n} \rightarrow^{*} \bar{u}$ ) in $X$. Evidently, $u=\bar{u}$ due to the continuous embedding and also $u=\bar{u} \in H$ due to the fact that $H$ is weakly (weakly*) closed. The fact that $u$ is a fixed point of $\mathcal{T}$ as well as its uniqueness follows exactly as in the proof of the classical Banach fixed-point theorem. 
3. Main results. We are going to formulate the main results here. Note that in comparison to the results proved in [4] or [1] we need less regular initial conditions. We use similar notation for studied problems, namely $D_{V E}$ denotes the problem with Dirichlet boundary conditions, $P_{V E}$ denotes periodic boundary conditions and $C_{V E}$ denotes the Cauchy problem.

First, let us formulate the results for the system (2). For the problems $D_{V E}$ and $P_{V E}$ we have

Theorem 3.1. Let $N=2,3, \Omega \in C^{2, \lambda}$ with $\lambda>0$ (for $D_{V E}$ ), $\mathbf{f} \in L^{2}\left(0, T, L^{2}(\Omega)\right) \cap$ $L^{q}\left(0, T, L^{p}(\Omega)\right)$ for certain $p>N$ and $q>1, \int_{\Omega} \mathbf{f} d x=\mathbf{0}$ (for $D_{V E}$ ). Let $\mathbf{F}_{0} \in$ $W^{1, p}(\Omega), \operatorname{div} \mathbf{F}_{0}=0$ and $\mathbf{v}_{0} \in W_{\text {div }}^{1,2}(\Omega)$. Then there exists $T^{*}>0$ such that on $\left(0, T^{*}\right)$ there exists a unique strong solution to the problem (2) with either Dirichlet or periodic boundary conditions such that $\mathbf{v} \in L^{2}\left(0, T^{*}, W^{2,2}(\Omega)\right) \cap L^{\infty}\left(0, T^{*}, W^{1,2}(\Omega)\right)$ and $\mathbf{F} \in L^{\infty}\left(0, T^{*}, W^{1, p}(\Omega)\right)$.

An analogous result holds for (3):

Theorem 3.2. Let $N=2,3, \Omega \in C^{2, \lambda}$ with $\lambda>0$ (for $D_{V E}$ ), $\mathbf{f} \in L^{2}\left(0, T, L^{2}(\Omega)\right) \cap$ $L^{q}\left(0, T, L^{p}(\Omega)\right)$ for certain $p>N$ and $q>1, \int_{\Omega} \mathbf{f} d x=\mathbf{0}$ (for $\left.P_{V E}\right)$. Let $\nabla \boldsymbol{\phi}_{0} \in W^{1, p}(\Omega)$ and $\mathbf{v}_{0} \in W_{\text {div }}^{1,2}(\Omega)$. Then there exists $T^{*}>0$ such that on $\left(0, T^{*}\right)$ there exists a unique strong solution to the problem (3) with either Dirichlet or periodic boundary conditions such that $\mathbf{v} \in L^{2}\left(0, T^{*}, W^{2,2}(\Omega)\right) \cap L^{\infty}\left(0, T^{*}, W^{1,2}(\Omega)\right)$ and $\nabla \phi \in L^{\infty}\left(0, T^{*}, W^{1, p}(\Omega)\right)$.

Concerning the Cauchy problem, it is not possible to expect $\mathbf{F}_{0}$ (or $\nabla \boldsymbol{\phi}_{0}$ ) to be integrable with any power. However, we may consider $\mathbf{H}_{0}=\mathbf{F}_{0}-\mathbf{I}$ and for $\mathbf{H}=\mathbf{F}-\mathbf{I}$ we get the following problem (recall that $\operatorname{div} \mathbf{F}=0$ )

$$
\left.\begin{array}{rl}
\frac{\partial \mathbf{v}}{\partial t}+\mathbf{v} \cdot \nabla \mathbf{v}-\mu \Delta \mathbf{v}+\nabla p & =\operatorname{div}\left(\mathbf{H H}^{T}\right)+\operatorname{div} \mathbf{H}^{T}+\mathbf{f} \\
\operatorname{div} \mathbf{v} & =0 \\
\frac{\partial \mathbf{H}}{\partial t}+\mathbf{v} \cdot \nabla \mathbf{H} & =\nabla \mathbf{v H}+\nabla \mathbf{v} \\
\mathbf{v}(0, \mathbf{x}) & =\mathbf{v}_{0}(\mathbf{x}) \quad \text { in } \mathbb{R}^{N}, \\
\mathbf{H}(0, \mathbf{x}) & =\mathbf{H}_{0}(\mathbf{x}) \quad \text { in } \mathbb{R}^{N} .
\end{array}\right\} \text { in }(0, T) \times \mathbb{R}^{N}
$$

Similarly, defining $\boldsymbol{\psi}(\mathbf{x})=\boldsymbol{\phi}(\mathbf{x})-\mathbf{x}$ we replace the problem (3) by

$$
\left.\begin{array}{cc}
\frac{\partial \mathbf{v}}{\partial t}+\mathbf{v} \cdot \nabla \mathbf{v}-\mu \Delta \mathbf{v}+ & \nabla p=-\sum_{i=1}^{N} \Delta \psi_{i} \nabla \psi_{i}-\Delta \boldsymbol{\psi}+\mathbf{f} \\
\operatorname{div} \mathbf{v}=0 & \\
\frac{\partial \boldsymbol{\psi}}{\partial t}+\mathbf{v} \cdot \nabla \boldsymbol{\psi}=-\mathbf{v} & \\
\mathbf{v}(0, \mathbf{x})=\mathbf{v}_{0}(\mathbf{x}) & \text { in } \mathbb{R}^{N} \\
\boldsymbol{\psi}(0, \mathbf{x})=\boldsymbol{\psi}_{0}(\mathbf{x}) & \text { in } \mathbb{R}^{N}
\end{array}\right\} \text { in }(0, T) \times \mathbb{R}^{N}
$$

We have

Theorem 3.3. Let $N=2,3, \mathbf{f} \in L^{2}\left(0, T, L^{2}\left(\mathbb{R}^{N}\right)\right) \cap L^{q}\left(0, T, L^{p}\left(\mathbb{R}^{N}\right)\right)$ for certain $p>$ $N$ and $q>1$. Let $\mathbf{H}_{0} \in W^{1, p}\left(\mathbb{R}^{N}\right) \cap W^{1,2}\left(\mathbb{R}^{N}\right)$, $\operatorname{div} \mathbf{H}_{0}=0$ and $\mathbf{v}_{0} \in W_{\text {div }}^{1,2}\left(\mathbb{R}^{N}\right)$. Then there exists $T^{*}>0$ such that on $\left(0, T^{*}\right)$ there exists a unique strong solution to 
the problem (6) such that $\mathbf{v} \in L^{2}\left(0, T^{*}, W^{2,2}\left(\mathbb{R}^{N}\right)\right) \cap L^{\infty}\left(0, T^{*}, W^{1,2}\left(\mathbb{R}^{N}\right)\right)$ and $\mathbf{H} \in$ $L^{\infty}\left(0, T^{*}, W^{1, p}\left(\mathbb{R}^{N}\right)\right)$.

Similarly for (7) we have

Theorem 3.4. Let $N=2,3, \mathbf{f} \in L^{2}\left(0, T, L^{2}\left(\mathbb{R}^{N}\right)\right) \cap L^{q}\left(0, T, L^{p}\left(\mathbb{R}^{N}\right)\right)$ for certain $p>N$ and $q>1$. Let $\nabla \boldsymbol{\psi}_{0} \in W^{1, p}\left(\mathbb{R}^{N}\right) \cap W^{1,2}\left(\mathbb{R}^{N}\right)$ and $\mathbf{v}_{0} \in W_{\text {div }}^{1,2}\left(\mathbb{R}^{N}\right)$. Then there exists $T^{*}>0$ such that on $\left(0, T^{*}\right)$ there exists a unique strong solution to the problem (7) such that $\mathbf{v} \in L^{2}\left(0, T^{*}, W^{2,2}\left(\mathbb{R}^{N}\right)\right) \cap L^{\infty}\left(0, T^{*}, W^{1,2}\left(\mathbb{R}^{N}\right)\right)$ and $\nabla \boldsymbol{\psi} \in L^{\infty}\left(0, T^{*}, W^{1, p}\left(\mathbb{R}^{N}\right)\right)$.

4. Proofs. In this section we are going to present the proofs of the results presented in the previous section. We start with Theorem 3.1.

Proof of Theorem 3.1. a) Let us consider first $N=2$. We take $2<p<3$ and we set $X=L^{\infty}\left(0, T, W^{1, p}(\Omega)\right), Y=L^{\infty}\left(0, T, L^{p}(\Omega)\right)$ and we will apply Lemma 2.5 on the mapping $\mathcal{T}=\mathcal{T}_{1} \circ \mathcal{T}_{2}: \overline{\mathbf{F}} \rightarrow \mathbf{F}$, where $\mathcal{T}_{2}: \overline{\mathbf{F}} \rightarrow \mathbf{v}$ and $\mathcal{T}_{1}: \mathbf{v} \rightarrow \mathbf{F}$ are the following mappings. $\mathcal{T}_{2}(\overline{\mathbf{F}})=\mathbf{v}$ which is a solution of the problem

$$
\left.\begin{array}{c}
\frac{\partial \mathbf{v}}{\partial t}+\mathbf{v} \cdot \nabla \mathbf{v}-\mu \Delta \mathbf{v}+\nabla p=\operatorname{div}\left(\overline{\mathbf{F}} \overline{\mathbf{F}}^{T}\right)+\mathbf{f} \\
\operatorname{div} \mathbf{v}=0 \\
\mathbf{v}(0, \mathbf{x})=\mathbf{v}_{0}(\mathbf{x}) \quad \text { in } \Omega
\end{array}\right\} \text { in }(0, T) \times \Omega
$$

and $\mathbf{v}=0$ on $\partial \Omega \times(0, T)$ for $D_{V E}, \mathbf{v}$ space periodic for $P_{V E}$ (then also $W^{1, p}(\Omega)$ and $L^{p}(\Omega)$ is replaced by $W_{\text {per }}^{1, p}(\Omega)$ and $\left.L_{\text {per }}^{p}(\Omega)\right)$. Further $\mathcal{T}_{1}(\mathbf{v})=\mathbf{F}$ which is a solution of the following problem

$$
\begin{aligned}
\frac{\partial \mathbf{F}}{\partial t}+\mathbf{v} \cdot \nabla \mathbf{F}=\nabla \mathbf{v F} \quad \text { in }(0, T) \times \Omega, \\
\mathbf{F}(0, \mathbf{x})=\mathbf{F}_{0}(\mathbf{x}) \quad \text { in } \Omega .
\end{aligned}
$$

We show that $\mathcal{T}$ maps sufficiently large balls in $L^{\infty}\left(0, T, W^{1, p}(\Omega)\right)$ into itself. Considering problem (9), i.e. the transport equation, for $\mathbf{v} \in L^{1}\left(0, T, W^{2, p}(\Omega)\right), p>2$ it is not difficult to prove the existence of a unique solution to (9) and to verify the validity of the following estimates

$$
\begin{gathered}
\|\mathbf{F}\|_{L^{\infty}\left(0, T, L^{q}\right)} \leq\left\|\mathbf{F}_{0}\right\|_{L^{q}} \exp \left(\int_{0}^{T}\|\nabla \mathbf{v}\|_{L^{\infty}} \mathrm{d} t\right), \quad 1 \leq q \leq \infty, \\
\|\nabla \mathbf{F}\|_{L^{\infty}\left(0, T, L^{p}\right)} \leq C\left(\left\|\nabla \mathbf{F}_{0}\right\|_{L^{p}}+\|\mathbf{F}\|_{L^{\infty}\left(0, T, L^{\infty}\right)} \int_{0}^{T}\left\|\nabla^{2} \mathbf{v}\right\|_{L^{p}} \mathrm{~d} t\right) \\
\times \exp \left(\int_{0}^{T}\|\nabla \mathbf{v}\|_{L^{\infty}} \mathrm{d} t\right) .
\end{gathered}
$$

Note that these estimates do not depend on the studied boundary value problems $\left(C_{V E}\right.$, $\left.D_{V E}, P_{V E}\right)$. The proof is based on multiplying the equations for $\mathbf{F}$ and $\nabla \mathbf{F}$ by $|\mathbf{F}|^{q-2} \mathbf{F}$ and $|\nabla \mathbf{F}|^{p-2} \nabla \mathbf{F}$, respectively, and integrating over $\Omega$, together with application of integration by parts and passing with $q$ to $\infty$.

Next, we need estimates of $\mathbf{v}$ in $L^{q}\left(0, T, W^{2, p}(\Omega)\right)$. As $\overline{\mathbf{F}} \in L^{\infty}\left(0, T, W^{1, p}(\Omega)\right)$, due to Lemma 2.3, there exists a unique solution to problem (8). Moreover, applying Lemma 2.1 
we have

$$
\left\|\nabla^{2} \mathbf{v}\right\|_{L^{q}\left(0, T, L^{p}\right)} \leq C\left(\left\|\mathbf{v}_{0}\right\|_{D_{p}^{1-1 / q, q}}+\left\|\operatorname{div}\left(\overline{\mathbf{F}} \overline{\mathbf{F}}^{T}\right)-\mathbf{v} \cdot \nabla \mathbf{v}+\mathbf{f}\right\|_{L^{q}\left(0, T, L^{p}\right)}\right) .
$$

Note that we may take $q$ sufficiently close to 1 and $p$ sufficiently close to 2 so that

$$
\left\|\mathbf{v}_{0}\right\|_{D_{p}^{1-1 / q, q}} \leq C\left\|\mathbf{v}_{0}\right\|_{W^{1,2}} .
$$

As $p>2$,

$$
\left\|\operatorname{div}\left(\overline{\mathbf{F}} \overline{\mathbf{F}}^{T}\right)\right\|_{L^{q}\left(0, T, L^{p}\right)} \leq T^{1 / q}\|\overline{\mathbf{F}}\|_{L^{\infty}\left(0, T, L^{\infty}\right)}\|\nabla \overline{\mathbf{F}}\|_{L^{\infty}\left(0, T, L^{p}\right)} \leq T^{1 / q} \cdot R^{2},
$$

where we denoted by $R$ the diameter of the ball $H$ in $L^{\infty}\left(0, T, W^{1, p}(\Omega)\right)$.

Finally, for $p<3$

$$
\|\mathbf{v} \cdot \nabla \mathbf{v}\|_{L^{p}} \leq\|\nabla \mathbf{v}\|_{L^{3}}\|\mathbf{v}\|_{L^{\frac{3 p}{3-p}}} \leq C\|\nabla \mathbf{v}\|_{W^{1,2}}^{1 / 3}\|\mathbf{v}\|_{W^{1,2}}^{5 / 3}
$$

and thus

$$
\|\mathbf{v} \cdot \nabla \mathbf{v}\|_{L^{q}\left(0, T, L^{p}\right)} \leq C T^{\frac{6-q}{6 q}}\|\nabla \mathbf{v}\|_{L^{2}\left(0, T, W^{1,2}\right)}^{1 / 3}\|\mathbf{v}\|_{L^{\infty}\left(0, T, W^{1,2}\right)}^{5 / 3} .
$$

We have to apply the energy method to estimate these two terms. First, multiplying (8) by $\mathbf{v}$ yields after standard operations

$$
\frac{1}{2} \frac{\mathrm{d}}{\mathrm{d} t}\|\mathbf{v}\|_{L^{2}}^{2}+\mu\|\nabla \mathbf{v}\|_{L^{2}}^{2} \leq \int_{\Omega}|\overline{\mathbf{F}}|^{2}|\nabla \mathbf{v}| \mathrm{d} \mathbf{x}+\int_{\Omega}|\mathbf{f} \cdot \mathbf{v}| \mathrm{d} \mathbf{x}
$$

which leads to

$$
\|\mathbf{v}\|_{L^{\infty}\left(0, T, L^{2}\right)}+\mu\|\nabla \mathbf{v}\|_{L^{2}\left(0, T, L^{2}\right)} \leq C\left(T^{1 / 2} R^{2}+\|\mathbf{f}\|_{L^{2}\left(0, T, L^{2}\right)}+\left\|\mathbf{v}_{0}\right\|_{L^{2}}\right) .
$$

Finally, we test the same equation with $-P \Delta \mathbf{v}$, where $P$ is the Leray projection (thus $P \Delta \mathbf{u}=\Delta \mathbf{u}$ in the case of periodic boundary conditions):

$$
\begin{aligned}
\frac{1}{2} \frac{\mathrm{d}}{\mathrm{d} t}\|\nabla \mathbf{v}\|_{L^{2}}^{2}+ & \mu\left\|\nabla^{2} \mathbf{v}\right\|_{L^{2}}^{2} \\
& \leq \int_{\Omega}\left|\operatorname{div}\left(\overline{\mathbf{F}} \overline{\mathbf{F}}^{T}\right) P \Delta \mathbf{v}\right| \mathrm{d} \mathbf{x}+\int_{\Omega}|(\mathbf{v} \cdot \nabla \mathbf{v}) \cdot P \Delta \mathbf{v}| \mathrm{d} \mathbf{x}+\int_{\Omega}|\mathbf{f} \cdot P \Delta \mathbf{v}| \mathrm{d} \mathbf{x} .
\end{aligned}
$$

Note that we used that $\left\|\nabla^{2} \mathbf{v}\right\|_{L^{2}} \leq\|P \Delta \mathbf{v}\|_{L^{2}}$ and in the case of periodic boundary conditions, the second term on the right hand side is zero. Using standard tools as Hölder's inequality and Sobolev imbedding theorem, we end up with

$$
\frac{\mathrm{d}}{\mathrm{d} t}\|\nabla \mathbf{v}\|_{L^{2}}^{2}+\mu\left\|\nabla^{2} \mathbf{v}\right\|_{L^{2}}^{2} \leq C(\mu)\left(1+T R^{4}\right)\left(\|\overline{\mathbf{F}}\|_{W^{1, p}}^{4}+\|\mathbf{f}\|_{L^{2}}^{2}+\|\nabla \mathbf{v}\|_{L^{2}}^{4}\right) .
$$

Thus

$$
\begin{aligned}
& \|\nabla \mathbf{v}\|_{L^{\infty}\left(0, T, L^{2}\right)}+\mu\left\|\nabla^{2} \mathbf{v}\right\|_{L^{2}\left(0, T, L^{2}\right)} \\
& \quad \leq C\left(1+T^{\frac{1}{2}} R^{2}\right) e^{\int_{0}^{T}\|\nabla \mathbf{v}\|_{L^{2}}^{2} \mathrm{~d} t}\left(T^{1 / 2}\|\overline{\mathbf{F}}\|_{L^{\infty}\left(0, T, W^{1, p}\right)}^{2}+\|\mathbf{f}\|_{L^{2}\left(0, T, L^{2}\right)}+\left\|\nabla \mathbf{v}_{0}\right\|_{L^{2}}\right)
\end{aligned}
$$

Plugging in estimates (18) and (16) into (15) and together with (13) and (14) we have

$$
\begin{aligned}
& \|\nabla \mathbf{v}\|_{L^{q}\left(0, T, W^{1, p}\right)} \leq C\left(\left\|\mathbf{v}_{0}\right\|_{W^{1,2}}+T^{1 / q} R^{2}+\|\mathbf{f}\|_{L^{q}\left(0, T, L^{p}\right)}\right. \\
& +C T^{\frac{6-q}{6 q}}\left[T R^{4}+\|\mathbf{f}\|_{L^{2}\left(0, T, L^{2}\right)}^{2}+\left\|\mathbf{v}_{0}\right\|_{L^{2}}^{2}+\left(1+T R^{4}\right)\right. \\
& \times e^{\left.\left.T R^{4}+\|\mathbf{f}\|_{L^{2}\left(0, T, L^{2}\right)}^{2}+\left\|\mathbf{v}_{0}\right\|_{L^{2}}^{2}\left(T\|\overline{\mathbf{F}}\|_{L^{\infty}\left(0, T, W^{1, p}\right)}^{4}+\|\mathbf{f}\|_{L^{2}\left(0, T, L^{2}\right)}^{2}+\left\|\nabla \mathbf{v}_{0}\right\|_{L^{2}}^{2}\right)\right]\right) .}
\end{aligned}
$$


Therefore, as $q>1$, we easily conclude from (10) and (11) that taking $R$ sufficiently large with respect to $\left\|\mathbf{F}_{0}\right\|_{W^{1, p}}$ there exists $T^{*}=T^{*}\left(q, p, R,\left\|\mathbf{v}_{0}\right\|_{W^{1,2}},\left\|\mathbf{F}_{0}\right\|_{W^{1, p}}, \Omega, \mathbf{f}\right)$ such that

$$
\|\overline{\mathbf{F}}\|_{L^{\infty}\left(0, T^{*}, W^{1, p}\right)} \leq R .
$$

The first part of Lemma 2.5 is satisfied.

Next, we have to verify that the mapping $\mathcal{T}$ is in fact a contraction on $L^{\infty}\left(0, T, L^{p}(\Omega)\right)$. To this end let us denote $\mathbf{v}^{i}=\mathcal{T}_{2}\left(\overline{\mathbf{F}}^{i}\right), i=1,2$ and $\mathbf{F}^{i}=\mathcal{T}_{1}\left(\mathbf{v}^{i}\right)$, thus $\mathbf{F}^{i}=\mathcal{T}\left(\overline{\mathbf{F}}^{i}\right)$. Moreover we denote $\mathbf{F}^{12}=\mathbf{F}^{1}-\mathbf{F}^{2}, \mathbf{v}^{12}=\mathbf{v}^{1}-\mathbf{v}^{2}$ and $\overline{\mathbf{F}}^{12}=\overline{\mathbf{F}}^{1}-\overline{\mathbf{F}}^{2}$. We have

$$
\begin{array}{ccc}
\frac{\partial \mathbf{F}^{12}}{\partial t}+\mathbf{v}^{1} \cdot \nabla \mathbf{F}^{12}+\mathbf{v}^{12} \cdot \nabla \mathbf{F}^{2} & =\nabla \mathbf{v}^{1} \mathbf{F}^{12}+\nabla \mathbf{v}^{12} \mathbf{F}^{2} & \text { in }(0, T) \times \Omega, \\
\mathbf{F}^{12}(0, \mathbf{x})=\mathbf{0} & \text { in } \Omega . &
\end{array}
$$

Similarly as in estimate (10) we get

$$
\begin{aligned}
& \frac{1}{p} \frac{\mathrm{d}}{\mathrm{d} t}\left\|\mathbf{F}^{12}\right\|_{L^{p}}^{p} \\
& \leq \int_{\Omega}\left|\mathbf{v}^{12}\left\|\nabla \mathbf{F}^{2}\right\| \mathbf{F}^{12}\right|^{p-1} \mathrm{~d} \mathbf{x}+\int_{\Omega}\left|\nabla \mathbf{v}^{1}\right|\left|\mathbf{F}^{12}\right|^{p} \mathrm{~d} \mathbf{x}+\int_{\Omega}\left|\nabla \mathbf{v}^{12}\left\|\mathbf{F}^{2}\right\| \mathbf{F}^{12}\right|^{p-1} \mathrm{~d} \mathbf{x} \\
& \quad \leq\left\|\mathbf{F}^{12}\right\|_{L^{p}}^{p-1}\left(\left\|\mathbf{v}^{12}\right\|_{L^{\infty}}\left\|\nabla \mathbf{F}^{2}\right\|_{L^{p}}+\left\|\nabla \mathbf{v}^{1}\right\|_{L^{\infty}}\left\|\mathbf{F}^{12}\right\|_{L^{p}}+\left\|\mathbf{F}^{2}\right\|_{L^{\infty}}\left\|\nabla \mathbf{v}^{12}\right\|_{L^{p}}\right),
\end{aligned}
$$

hence

$$
\begin{aligned}
& \left\|\mathbf{F}^{12}\right\|_{L^{\infty}\left(0, T, L^{p}\right)} \\
& \leq C e^{\int_{0}^{T}\left\|\nabla \mathbf{v}^{1}\right\|_{L^{\infty}} \mathrm{d} t} \int_{0}^{T}\left(\left\|\mathbf{v}^{12}\right\|_{L^{\infty}}\left\|\nabla \mathbf{F}^{2}\right\|_{L^{p}}+\left\|\mathbf{F}^{2}\right\|_{L^{\infty}}\left\|\nabla \mathbf{v}^{12}\right\|_{L^{p}}\right) \mathrm{d} t \\
& \leq C_{1} R \int_{0}^{T}\left\|\mathbf{v}^{12}\right\|_{W^{1, p}} \mathrm{~d} t .
\end{aligned}
$$

It remains to estimate the difference $\mathbf{v}^{12}$ in $L^{p}\left(0, T, W^{1, p}(\Omega)\right.$ ). We have (denoting by $p^{12}$ the difference of corresponding pressures)

$$
\left.\begin{array}{c}
\frac{\partial \mathbf{v}^{12}}{\partial t}-\mu \Delta \mathbf{v}^{12}+\nabla p^{12}=\operatorname{div}\left(\overline{\mathbf{F}}^{12} \overline{\mathbf{F}}^{1 T}+\overline{\mathbf{F}}^{2} \overline{\mathbf{F}}^{12 T}\right)- \\
\mathbf{v}^{1} \cdot \nabla \mathbf{v}^{12}-\mathbf{v}^{12} \cdot \nabla \mathbf{v}^{2} \\
\operatorname{div} \mathbf{v}^{12}=0 \\
\mathbf{v}^{12}(0, \mathbf{x})=\mathbf{0} \quad \text { in } \Omega
\end{array}\right\} \text { in }(0, T) \times \Omega
$$

together with corresponding boundary conditions (Dirichlet or periodic ones). Applying Lemma 2.2 we immediately see that

$$
\begin{aligned}
\left\|\nabla \mathbf{v}^{12}\right\|_{L^{p}\left(0, T, L^{p}\right)} \leq C\left(\left\|\overline{\mathbf{F}}^{12} \overline{\mathbf{F}}^{1 T}+\overline{\mathbf{F}}^{2} \overline{\mathbf{F}}^{12 T}-\mathbf{v}^{1} \mathbf{v}^{12}-\mathbf{v}^{12} \mathbf{v}^{2}\right\|_{L^{p}\left(0, T, L^{p}\right)}\right) \\
\leq C_{1}\left(\sum_{i=1}^{2}\left\|\mathbf{v}^{i} \mathbf{v}^{12}\right\|_{L^{p}\left(0, T, L^{p}\right)}+\sum_{i=1}^{2}\left\|\overline{\mathbf{F}}^{i} \overline{\mathbf{F}}^{12 T}\right\|_{L^{p}\left(0, T, L^{p}\right)}\right) .
\end{aligned}
$$

Evidently

$$
\left\|\overline{\mathbf{F}}^{i} \overline{\mathbf{F}}^{12 T}\right\|_{L^{p}\left(0, T, L^{p}\right)} \leq T^{1 / p}\left\|\overline{\mathbf{F}}^{i}\right\|_{L^{\infty}\left(0, T, L^{\infty}\right)}\left\|\overline{\mathbf{F}}^{12}\right\|_{L^{\infty}\left(0, T, L^{p}\right)} \leq R T^{1 / p}\left\|\overline{\mathbf{F}}^{12}\right\|_{L^{\infty}\left(0, T, L^{p}\right)} .
$$


In the other term we must be more careful:

$$
\begin{array}{r}
\left\|\mathbf{v}^{i} \mathbf{v}^{12}\right\|_{L^{p}\left(0, T, L^{p}\right)} \leq C\left\|\mathbf{v}^{i}\right\|_{L^{\infty}\left(0, T, W^{1,2}\right)}\left(\int_{0}^{T}\left\|\nabla \mathbf{v}^{12}\right\|_{L^{p}}^{\frac{p^{2}}{6(p-1)}}\left\|\mathbf{v}^{12}\right\|_{L^{2}}^{\frac{p(5 p-6)}{6(p-1)}} \mathrm{d} t\right)^{1 / p} \\
\leq C T^{\frac{5 p-6}{6 p-6} \frac{1}{p}}\left\|\mathbf{v}^{i}\right\|_{L^{\infty}\left(0, T, W^{1,2}\right)}\left(\left\|\nabla \mathbf{v}^{12}\right\|_{L^{p}\left(0, T, L^{p}\right)}+\left\|\mathbf{v}^{12}\right\|_{L^{\infty}\left(0, T, L^{2}\right)}\right) .
\end{array}
$$

Taking $T$ sufficiently small, the first term can be absorbed into the left-hand side of (23), while for the second term we use the energy method. We multiply (22) by $\mathbf{v}^{12}$ and integrate over $\Omega$ :

$$
\begin{gathered}
\frac{1}{2} \frac{\mathrm{d}}{\mathrm{d} t}\left\|\mathbf{v}^{12}\right\|_{L^{2}}^{2}+\mu\left\|\nabla \mathbf{v}^{12}\right\|_{L^{2}}^{2} \leq \int_{\Omega}\left|\mathbf{v}^{12}\right|^{2}\left|\nabla \mathbf{v}^{2}\right| \mathrm{d} \mathbf{x}+\int_{\Omega}\left|\overline{\mathbf{F}}^{12}\right|\left(\left|\overline{\mathbf{F}}^{1}\right|+\left|\overline{\mathbf{F}}^{2}\right|\right)\left|\nabla \mathbf{v}^{12}\right| \mathrm{d} \mathbf{x} \leq \\
\leq\left\|\mathbf{v}^{12}\right\|_{L^{2}}\left\|\nabla \mathbf{v}^{12}\right\|_{L^{2}}\left\|\nabla \mathbf{v}^{2}\right\|_{L^{2}}+\left\|\nabla \mathbf{v}^{12}\right\|_{L^{2}}\left\|\overline{\mathbf{F}}^{12}\right\|_{L^{p}}\left(\left\|\overline{\mathbf{F}}^{1}\right\|_{W^{1, p}}+\left\|\overline{\mathbf{F}}^{2}\right\|_{W^{1, p}}\right)
\end{gathered}
$$

Therefore

$$
\frac{\mathrm{d}}{\mathrm{d} t}\left\|\mathbf{v}^{12}\right\|_{L^{2}}^{2}+\mu\left\|\nabla \mathbf{v}^{12}\right\|_{L^{2}}^{2} \leq C(\mu)\left(\left\|\mathbf{v}^{12}\right\|_{L^{2}}^{2}\left\|\nabla \mathbf{v}^{2}\right\|_{L^{2}}^{2}+2 R^{2}\left\|\overline{\mathbf{F}}^{12}\right\|_{L^{p}}^{2}\right)
$$

which implies

$$
\left\|\mathbf{v}^{12}\right\|_{L^{\infty}\left(0, T, L^{2}\right)}+\mu\left\|\nabla \mathbf{v}^{12}\right\|_{L^{2}\left(0, T, L^{2}\right)} \leq C R T^{1 / 2} e^{\int_{0}^{T}\left\|\nabla \mathbf{v}^{2}\right\|_{L^{2}}^{2} \mathrm{~d} t}\left\|\overline{\mathbf{F}}^{12}\right\|_{L^{\infty}\left(0, T, L^{p}\right)} .
$$

Thus, estimates (23)-(26) imply

$$
\begin{aligned}
& \left\|\nabla \mathbf{v}^{12}\right\|_{L^{p}\left(0, T, L^{p}\right)} \leq \\
& \quad \leq C_{1} R T^{1 / p}\left\|\overline{\mathbf{F}}^{12}\right\|_{L^{\infty}\left(0, T, L^{p}\right)}+C_{2}\left(\mathbf{F}_{0}, R, \mathbf{f}, \mathbf{v}_{0}\right) T^{\frac{1}{p} \frac{5 p-6}{6 p-6}+\frac{1}{2}}\left\|\overline{\mathbf{F}}^{12}\right\|_{L^{\infty}\left(0, T, L^{p}\right)} .
\end{aligned}
$$

From (27) and (21) we get

$$
\left\|\mathbf{F}^{12}\right\|_{L^{\infty}\left(0, T, L^{p}\right)} \leq C\left(R, \mathbf{f}, \mu, \mathbf{F}_{0}, \mathbf{v}_{0}\right) T\left\|\overline{\mathbf{F}}^{12}\right\|_{L^{\infty}\left(0, T, L^{p}\right)}
$$

which yields for $T$ sufficiently small that $\mathcal{T}$ is a contraction in $L^{\infty}\left(0, T, L^{p}(\Omega)\right)$. The theorem for $N=2$ is proved.

b) The case $N=3$. The proof works basically similarly, with $p>3$. There is one main difference which reflects the fundamental difference between the regularity of solutions to the two- and three dimensional Navier-Stokes equations, see below. Except for this, there are only a few changes connected with interpolation inequalities. For $p<6$,

$$
\|\mathbf{v} \cdot \nabla \mathbf{v}\|_{L^{p}} \leq\|\nabla \mathbf{v}\|_{L^{p}}\|\mathbf{v}\|_{L^{\infty}} \leq C\|\nabla \mathbf{v}\|_{W^{1,2}}^{\frac{2 p-3}{p}}\|\nabla \mathbf{v}\|_{L^{2}}^{\frac{3}{p}}
$$

and the estimate (15) is replaced by

$$
\|\mathbf{v} \cdot \nabla \mathbf{v}\|_{L^{q}\left(0, T, L^{p}\right)} \leq C T^{\frac{2 p-q(2 p-3)}{2 p q}}\|\nabla \mathbf{v}\|_{L^{2}\left(0, T, W^{1,2}\right)}^{\frac{2 p-3}{p}}\|\mathbf{v}\|_{L^{\infty}\left(0, T, W^{1,2}\right)}^{\frac{3}{p}} .
$$

Note that we may always take $1<q<\frac{2 p}{2 p-3}$ and the power at $T$ is positive. The main difference is in estimate (18). While in the $2 \mathrm{D}$ case we were able to get the estimates immediately (note that we have global-in-time existence of strong solutions to the NavierStokes equations), we have now instead of (17)

$$
\frac{\mathrm{d}}{\mathrm{d} t}\|\nabla \mathbf{v}\|_{L^{2}}^{2}+\mu\left\|\nabla^{2} \mathbf{v}\right\|_{L^{2}}^{2} \leq C(\mu)\left(\|\overline{\mathbf{F}}\|_{W^{1, p}}^{4}+\|\mathbf{f}\|_{L^{2}}^{2}+\|\nabla \mathbf{v}\|_{L^{2}}^{6}\right)
$$


from which one can only deduce that there is $T^{*}=T^{*}\left(\|\overline{\mathbf{F}}\|_{L^{\infty}\left(0, T, W^{1, p}\right)}, \mathbf{f}, \mathbf{v}_{0}\right)>0$ and $C^{*}=C^{*}\left(T^{*},\|\overline{\mathbf{F}}\|_{L^{\infty}\left(0, T, W^{1, p}\right)}, \mathbf{f}, \mathbf{v}_{0}\right)$ such that there is a strong solution to (8) in $3 \mathrm{D}$ on $\left(0, T^{*}\right)$ with

$$
\|\nabla \mathbf{v}\|_{L^{\infty}\left(0, T^{*}, L^{2}\right)}+\left\|\nabla^{2} \mathbf{v}\right\|_{L^{2}\left(0, T^{*}, L^{2}\right)} \leq C^{*} .
$$

Estimate (25) is replaced by

$$
\begin{aligned}
\left\|\mathbf{v}^{i} \mathbf{v}^{12}\right\|_{L^{p}\left(0, T, L^{p}\right)} & \leq C\left(\int_{0}^{T}\left\|\mathbf{v}^{i}\right\|_{L^{6}}^{p}\left\|\nabla \mathbf{v}^{12}\right\|_{L^{p}}^{p^{\frac{4 p-6}{p}}}\left\|\mathbf{v}^{12}\right\|_{L^{2}}^{\frac{p^{2}}{5 p-6}} \mathrm{~d} t\right)^{1 / p} \\
\leq & C T^{\frac{1}{5 p-6}}\left\|\mathbf{v}^{i}\right\|_{L^{\infty}\left(0, T, W^{1,2}\right)}\left(\left\|\nabla \mathbf{v}^{12}\right\|_{L^{p}\left(0, T, L^{p}\right)}+\left\|\mathbf{v}^{12}\right\|_{L^{\infty}\left(0, T, L^{2}\right)}\right) .
\end{aligned}
$$

Instead of (26) we get

$$
\left\|\mathbf{v}^{12}\right\|_{L^{\infty}\left(0, T, L^{2}\right)}+\mu\left\|\nabla \mathbf{v}^{12}\right\|_{L^{2}\left(0, T, L^{2}\right)} \leq C R T^{1 / 2} e^{\int_{0}^{T}\left\|\nabla \mathbf{v}^{2}\right\|_{L^{2}}^{4} \mathrm{~d} t}\left\|\overline{\mathbf{F}}^{12}\right\|_{L^{\infty}\left(0, T, L^{p}\right)}
$$

and instead of (27) we have

$$
\left\|\nabla \mathbf{v}^{12}\right\|_{L^{p}\left(0, T, L^{p}\right)} \leq C_{1} R T^{1 / p}\left\|\overline{\mathbf{F}}^{12}\right\|_{L^{\infty}\left(0, T, L^{p}\right)}+C_{2} T^{\frac{5 p-4}{10 p-12}}\left\|\overline{\mathbf{F}}^{12}\right\|_{L^{\infty}\left(0, T, L^{p}\right)} .
$$

Thus, provided $T \leq T^{*}$ is sufficiently small and $R$ sufficiently large, we get that the mapping $\mathcal{T}$ maps balls in $L^{\infty}\left(0, T, W^{1, p}(\Omega)\right)$ into itself and it is a contraction in the space $L^{\infty}\left(0, T, L^{p}(\Omega)\right)$. This finishes the proof of Theorem 3.1 for $N=3$.

Proof of Theorem 3.2. First note that

$$
\frac{\partial}{\partial t} \nabla \boldsymbol{\phi}+\mathbf{v} \cdot \nabla \nabla \boldsymbol{\phi}+\nabla \mathbf{v} \nabla \boldsymbol{\phi}=\mathbf{0}
$$

and

$$
\frac{\partial}{\partial t} \nabla^{2} \boldsymbol{\phi}+\mathbf{v} \cdot \nabla \nabla^{2} \boldsymbol{\phi}+\nabla^{2} \mathbf{v} \nabla \boldsymbol{\phi}=\mathbf{0}
$$

thus, in the estimates we need, $\nabla \phi \sim \mathbf{F}$ and $\nabla^{2} \phi \sim \nabla \mathbf{F}$. The proof works now more or less in the same way as in Theorem 3.1, with only one exception. It is impossible to write $\sum_{i=1}^{2} \nabla \phi_{i} \Delta \phi_{i}$ as the divergence of some quantity which behaves like $|\nabla \phi|^{2}$. Therefore in (23) the second term is replaced by (again, we denote $\phi^{12}=\phi^{1}-\phi^{2}$ )

$$
I \equiv\left\|\nabla \phi^{i} \Delta \phi^{12}\right\|_{L^{p}\left(0, T, W^{-1, p}\right)}+\left\|\Delta \phi^{i} \nabla \phi^{12}\right\|_{L^{p}\left(0, T, W^{-1, p}\right)} .
$$

In $2 \mathrm{D}$ we have

$$
\begin{aligned}
& I \leq\left\|\nabla \phi^{i} \nabla \phi^{12}\right\|_{L^{p}\left(0, T, L^{p}\right)}+\left\|\nabla^{2} \phi^{i} \nabla \phi^{12}\right\|_{L^{p}\left(0, T, L^{\frac{2 p}{p+2}}\right)} \\
& \leq C T^{1 / p}\left\|\nabla \phi^{12}\right\|_{L^{\infty}\left(0, T, L^{p}\right)}\left(\left\|\nabla \phi^{i}\right\|_{L^{\infty}\left(0, T, L^{\infty}\right)}+\left\|\nabla^{2} \phi^{i}\right\|_{L^{\infty}\left(0, T, L^{2}\right)}\right) \\
& \quad \leq C T^{1 / p}\left\|\nabla \phi^{12}\right\|_{L^{\infty}\left(0, T, L^{p}\right)}\left\|\nabla \phi^{i}\right\|_{L^{\infty}\left(0, T, W^{1, p}\right)}
\end{aligned}
$$

and in 3D

$$
\begin{aligned}
I \leq\left\|\nabla \phi^{i} \nabla \phi^{12}\right\|_{L^{p}\left(0, T, L^{p}\right)}+\left\|\nabla^{2} \phi^{i} \nabla \phi^{12}\right\|_{L^{p}\left(0, T, L^{\frac{3 p}{p+3}}\right)} \\
\leq C T^{1 / p}\left\|\nabla \phi^{12}\right\|_{L^{\infty}\left(0, T, L^{p}\right)}\left(\left\|\nabla \phi^{i}\right\|_{L^{\infty}\left(0, T, L^{\infty}\right)}+\left\|\nabla^{2} \phi^{i}\right\|_{L^{\infty}\left(0, T, L^{3}\right)}\right) \\
\quad \leq C T^{1 / p}\left\|\nabla \phi^{12}\right\|_{L^{\infty}\left(0, T, L^{p}\right)}\left\|\nabla \phi^{i}\right\|_{L^{\infty}\left(0, T, W^{1, p}\right)} .
\end{aligned}
$$

The rest follows exactly as in the proof of Theorem 3.1. 
Proof of Theorems 3.3 and 3.4. Due to the presence of linear terms in the right-hand side of (6) and (7) we have to replace the spaces. We set

$$
\begin{aligned}
& X=L^{\infty}\left(0, T, W^{1, p}\left(\mathbb{R}^{N}\right) \cap W^{1,2}\left(\mathbb{R}^{N}\right)\right) . \\
& Y=L^{\infty}\left(0, T, L^{p}\left(\mathbb{R}^{N}\right) \cap L^{2}\left(\mathbb{R}^{N}\right)\right), \quad p>N .
\end{aligned}
$$

We additionally need estimates of $\mathbf{H}$ in $L^{\infty}\left(0, T, W^{1,2}\left(\mathbb{R}^{N}\right)\right)$ and $\mathbf{H}^{12}=\mathbf{H}^{1}-\mathbf{H}^{2}$ in $L^{\infty}\left(0, T, L^{2}\left(\mathbb{R}^{N}\right)\right)$, similarly for $\nabla \boldsymbol{\psi}$. However, these estimates can be easily deduced from the corresponding estimates with $p>N$. These estimates are necessary as in (6) and in (7) linear terms of the type $\operatorname{div} \mathbf{H}^{T}$ and $\Delta \boldsymbol{\psi}$ appear additionally. We can now follow step by step the proof of Theorems 3.1 and 3.2 .

Acknowledgements. The first author was supported by the project LC06052 (Jindřich Nečas Center for Mathematical Modeling) and by the Charles University Grant Agency under Contract 2509/2007. The work of the second author is a part of the research project MSM 0021620839 financed by MSMT and partly supported by the grant of the Czech Science Foundation No. 201/06/0352, by the Charles University Grant Agency under Contract 2509/2007 and by the project LC06052 (Jindřich Nečas Center for Mathematical Modeling).

\section{References}

[1] Y. Chen and P. Zhang, The global existence of small solutions to the incompressible viscoelastic fluid system in 2 and 3 space dimensions, Comm. Part. Diff. Eq. 31 (2006), 1793-1810.

[2] M. Giga, Y. Giga and H. Sohr, $L^{p}$ estimates for the Stokes system, in: Functional Analysis and Related Topics (Kyoto, 1991), Lecture Notes in Math. 1540, Springer, Berlin, 1993, $55-67$.

[3] Y. Giga and H. Sohr, Abstract $L^{p}$ estimates for the Cauchy problem with applications to the Navier-Stokes equations in exterior domains, J. Funct. Anal. 102 (1991), 72-94.

[4] F. Lin, C. Liu and P. Zhang, On hydrodynamics of viscoelastic fluids, Comm. Pure Appl. Math. 58 (2005), 1437-1471.

[5] P.-L. Lions and N. Masmoudi, Global solutions for some Oldroyd models of non-Newtonian flows, Chinese Ann. Math. Ser. B 21 (2000), 131-146.

[6] C. Liu and N. J. Walkington, An Eulerian description of fluids containing visco-elastic particles, Arch. Ration. Mech. Anal. 159 (2001), 229-252.

[7] N. Masmoudi, Well posedness for the FENE dumbbell model of polymeric flows, preprint, New York University, 2007.

[8] R. Temam, Navier-Stokes Equations. Theory and Numerical Analysis, Reprint of the 1984 edition, AMS Chelsea Publ., Providence, RI, 2001. 